\title{
ANALYSIS OF COUNT DATA USING POWER VARIANCE FUNCTION
}

\author{
Eiji Nakashima*
}

\begin{abstract}
This paper considers two estimation methods for count data with a power variance function. One is the maximum likelihood method based on a negative binomial model with a power variance function which is not the standard application of a negative-binomial model. Another is the quasilikelihood/pseudolikelihood (QL/PL) estimating equation method. The $\mathrm{QL} / \mathrm{PL}$ method is a robust method and is applicable to a more general exponential dispersion model with a power variance function. The asymptotic efficiency of the QL/PL estimates were calculated relative to the maximum likelihood estimates, and demonstrated that the mean parameter estimate is approximately fully efficient. If the power parameter of the variance is close to one, then the efficiency of the power parameter of the variance is close to one. It was also found that, in a negative-binomial model with power variance function, mean parameter estimates and variance parameter estimates are approximately asymptotically independnet. An example of data analysis using power variance function is given.
\end{abstract}

Key words: extra-Poisson variation; estimating equation; quasilikelihood; pseudolikelihood; negative-binomial; power variance function; efficiency; titration

\section{Introduction}

Studies on distributions with a power variance function have recently been conducted (Jorgensen 1987; Nishii 1992). Jorgensen investigated a family of exponential dispersion models with a power variance function with power $r\left(\mathrm{ED}^{(r)}-\mathrm{PVF}\right)$. In general, estimation by the exact likelihood method is hard to carry out since the form of the distribution is complicated. However, $\mathrm{ED}^{(r)}-\mathrm{PVF}$ is a generalized linear model (GLM) family when $r$ is fixed. Therefore, we can implement an analysis under $\mathrm{ED}^{(r)}-\mathrm{PVF}$ by the quasilikelihood method using a GLIM program (Baker and Nelder 1978).

Carroll and Ruppert (1982) suggested the use of pseudolikelihood for variance function estimation, and Davidian and Carroll $(1987,1988)$ used power variance function as an example of the use of pseudolikelihood. In this paper, an analysis of count data, the variance of which is a power of the mean is discussed.

If we apply a likelihood method to count data which has a power variance function, it would be preferable to use a gamma mixture of a Poisson (negativebinomial) model because of its mathematical tractability and flexibility. When we model the gamma parameters as appropriate, a model with a power variance function can be derived. While the quasilikelihood method is a robust method

Received July, 1994. Revised October 1994, March 1995. Accepted March, 1995

* Department of Statistics, Radiation Effects Research Foundation, Hijiyama Park 5-2, Minamiku, Hiroshima 732, Japan 
without assumption of higher order moments, it is also flexible.

In this paper, we consider an analysis of count data, $y_{i}(i=1, \ldots, N)$, with the following mean and variance model,

$$
\left\{\begin{array}{l}
E\left(y_{i}\right)=\mu_{i}=x_{i} \beta \\
\operatorname{Var}\left(y_{i}\right)=V i\left(\mu_{i}\right)=\phi \mu_{i}^{\lambda} .
\end{array}\right.
$$

The variance function is a power of the mean.

In section 2, we propose a negative binomial model with power variance function (NB-PVF). In section 3, we describe a quasilikelihood/pseudolikelihood (QL/PL) estimating equation method (Breslow 1990b). In section 4, we calculate the asymptotic relative efficiency of the estimate from the QL/PL method relative to the NB-PVF maximum likelihood estimate. In section 5, we give an example of analysis of count data that the variance is proportional to a power of the mean, which Breslow (1990a) has analyzed.

\section{Negative-binomial model with power variance function}

When a likelihood model is applied to data, it is usually convenient to apply a tractable model. Thus, we try here to apply a negative-binomial model with power variance function to the data.

Now let $y_{i}(i=1, \ldots, N)$ be a Poisson observation with index parameter $\rho_{i}$, then the probability of $y_{i}$ given $\rho_{i}$ is given by,

$$
h\left(y_{i} \mid \rho_{i}\right)=\frac{\rho_{i}^{y_{i}} e^{-\rho_{i}}}{y_{i} !} \text {. }
$$

We further assume that $\rho_{i}$ has a gamma prior $g_{i}$,

$$
g_{i}\left(\rho_{i}\right)=\frac{a_{i}\left(a_{i} \rho_{i}\right)^{b_{i}-1} e^{-a_{i} \rho_{i}}}{\Gamma\left(b_{i}\right)} .
$$

Then, we get a posterior distribution of $y_{i}$,

$$
\begin{aligned}
f\left(y_{i}\right) & =\int_{0}^{\infty} h\left(y_{i} \mid \rho_{i}\right) g_{i}\left(\rho_{i}\right) d \rho_{i} \\
& =\frac{\Gamma\left(y_{i}+b_{i}\right)}{y_{i} ! \Gamma\left(b_{i}\right)} \frac{a_{i}^{b_{i}}}{\left(a_{i}+1\right)^{\boldsymbol{y}_{i}+o_{i}}} .
\end{aligned}
$$

We have thus obtained a negative-binomial model. Fisher $(1941,1953)$ suggested the use of maximum likelihood estimate for the negative-binomial model. Jorgensen (1987) extended the negative-binomial model to the negative-multinomial model.

Then, $y_{i}$ has the following mean and variance,

$$
\left\{\begin{array}{l}
E\left(y_{i}\right)=\frac{b_{i}}{a_{i}}, \\
\operatorname{Var}\left(y_{i}\right)=\frac{b_{i}}{a_{i}}+\frac{b_{i}}{a_{i}^{2}} .
\end{array}\right.
$$

Now we let the mean model and the variance model be

$$
\left\{\begin{array}{l}
E\left(y_{i}\right)=\mu_{i} \\
\operatorname{Var}\left(y_{i}\right)=\phi \mu_{i}^{i}
\end{array}\right.
$$


where $\mu_{i}=\mu\left(x_{i}, \beta\right)$. The parameter $\phi$ is an extended coefficient of variation in count data and the parameter $\lambda$ is some measure of departure from Poisson data since at $\phi=1$, the distribution tends to Poisson $(\mu)$ when $\lambda$ tends to one. Then, from (5) and (6), we get

$$
\left\{\begin{array}{l}
a_{i}=1 /\left(\phi \mu_{i}^{i-1}-1\right), \\
b_{i}=\mu_{i} /\left(\phi \mu_{i}^{i-1}-1\right) .
\end{array}\right.
$$

A negative-binomial model with a power variance function (NB-PVF) is thus obtained. That is, the mean variance relationship (6) is embedded into a negative-binomial model by equations (7). Note this model includes the Poisson model as a special case. Also note that the score equation of the mean parameters does not become a quasilikelihood estimating equation as shown later.

In a standard negative-binomial application in which $\operatorname{Var}(y)=\mu+\alpha \mu^{2}$, the score equation for the mean parameters becomes a quasilikelihood estimating equation. Then, in a standard negative-binomial model, even if the variance model is misspecified, the mean parameter estimates are consistent and asymptotically independent of the dispersion parameters since the mean parameters and the dispersion parameters are orthogonal (Cox and Reid 1987). The application of this standard negative-binomial model is shown in Lawless (1987). In NB-PVF, when the variance function is misspecified even if the mean parameter is correctly specified, we have to keep in mind that the mean parameter estimates may have an asymptotic bias like a beta-binomial model (Kupper et al. 1986; Yamamoto and Yanagimoto 1988).

From the above models (4) and (7), we can calculate efficient score for $\beta$ and $\theta=(\log (\phi), \lambda)=(\tau, \lambda)$, and the second derivative of the likelihood with respect to $\beta$ and $\theta$. We can implement the Newton-Raphson method to get the maximum likelihood estimates of the parameters $(\beta, \tau, \lambda)$.

The $\log$ likelihood of the NB-PVF is given by

$$
\begin{aligned}
l(\beta, \tau, \lambda)= & \sum_{i=1}^{N} l_{i}(\beta, \tau, \lambda)=\sum_{i=1}^{N}\left\{\sum_{j=0}^{y_{i}-1} \log \left(j+\mu_{i} / c_{i}\right)\right. \\
& \left.-\left(\mu_{i} / c_{i}\right) \log \left(1+c_{i}\right)+y_{i} \log \left(c_{i} /\left(1+c_{i}\right)\right)\right\},
\end{aligned}
$$

where $c_{i}=\exp (\tau) \mu_{i}^{\lambda-1}-1$ and $\mu_{i}=x_{i} \beta$. The score is given by

$$
\begin{aligned}
\frac{\partial l}{\partial \beta} & =\sum_{i=1}^{N} \frac{\partial l_{i}}{\partial \beta} \\
& =\sum_{i=1}^{N}\left[\left\{\frac{1}{c_{i}}-\frac{(\lambda-1)\left(c_{i}+1\right)}{c_{i}^{2}}\right\}\left\{\sum_{j=0}^{y_{i}-1} \frac{1}{j+\mu_{i} / c_{i}}-\log \left(1+c_{i}\right)\right\}+(\lambda-1) \frac{y_{i}-\mu_{i}}{c_{i} \mu_{i}}\right] x_{i}, \\
\frac{\partial l}{\partial \tau} & =\sum_{i=1}^{N} \frac{\partial l_{i}}{\partial \tau} \\
& =\sum_{i=1}^{N}\left[\left\{\log \left(1+c_{i}\right)-\sum_{j=0}^{y_{i}-1} \frac{1}{j+\mu_{i} / c_{i}}\right\} \frac{\mu_{i}\left(1+c_{i}\right)}{c_{i}^{2}}+\frac{y_{i}-\mu_{i}}{c_{i}}\right],
\end{aligned}
$$

and

$$
\frac{\partial l}{\partial \lambda}=\sum_{i=1}^{N} \frac{\partial l_{i}}{\partial \lambda}=\sum_{i=1}^{N} \frac{\partial l_{i}}{\partial \tau} \log \left(\mu_{i}\right) \text {. }
$$


The second derivatives of the likelihood are

$$
\begin{aligned}
\frac{\partial^{2} l}{\partial \beta^{2}}= & \sum_{i=1}^{N} \frac{\partial^{2} l_{i}}{\partial \beta^{2}}=\sum_{i=1}^{N}\left[\left\{\lambda-2+\frac{2(\lambda-1)}{c_{i}}\right\}\left\{\sum_{j=0}^{y_{i}-1} \frac{1}{j+\mu_{i} / c_{i}}-\log \left(1+c_{i}\right)\right\} \frac{(\lambda-1)\left(c_{i}+1\right)}{\mu_{i} c_{i}^{2}}\right. \\
& -\left\{\frac{1}{c_{i}}-\frac{(\lambda-1)\left(c_{i}+1\right)}{c_{i}^{2}}\right\}\left\{\sum_{j=0}^{y_{i}-1} \frac{1}{\left(j+\mu_{i} / c_{i}\right)^{2}}\left(\frac{1}{c_{i}}-\frac{(\lambda-1)\left(c_{i}+1\right)}{c_{i}^{2}}\right)\right. \\
& \left.\left.+\frac{\lambda-1}{\mu_{i}}\right\}-\frac{\lambda-1}{c_{i} \mu_{i}}\right] x_{i} x_{i}^{T}, \\
\frac{\partial^{2} l}{\partial \beta \partial \tau}= & \sum_{i=1}^{N} \frac{\partial^{2} l_{i}}{\partial \beta \partial \tau}=\sum_{i=1}^{N}\left[\left\{\sum_{j=0}^{y_{i}-1} \frac{1}{\left(j+\mu_{i} / c_{i}\right)^{2}} \frac{\mu_{i}\left(c_{i}+1\right)}{c_{i}^{2}}-1\right\}\left\{\frac{1}{c_{i}}-\frac{(\lambda-1)\left(c_{i}+1\right)}{c_{i}^{2}}\right\}\right. \\
& \left.+\left\{\sum_{j=0}^{y_{i}-1} \frac{1}{j+\mu_{i} / c_{i}}-\log \left(1+c_{i}\right)\right\}\left\{\frac{2(\lambda-1)\left(c_{i}+1\right)}{c_{i}}-\lambda\right\} \frac{c_{i}+1}{c_{i}^{2}}\right] x_{i}, \\
\frac{\partial^{2} l}{\partial \beta \partial \lambda}= & \sum_{i=1}^{N} \frac{\partial^{2} l_{i}}{\partial \beta \partial \tau} \log \left(\mu_{i}\right), \\
\frac{\partial^{2} l}{\partial \tau^{2}}= & \sum_{i=1}^{N} \frac{\partial^{2} l_{i}}{\partial \tau^{2}}=\sum_{i=1}^{N}\left[\left\{1-\sum_{j=0}^{y_{i}-1} \frac{1}{\left(j+\mu_{i} / c_{i}\right)^{2}} \frac{\mu_{i}\left(c_{i}+1\right)}{c_{i}^{2}}\right\} \frac{\mu_{i}\left(c_{i}+1\right)}{c_{i}^{2}}\right. \\
& \left.-\left\{\log \left(c_{i}+1\right)-\sum_{j=0}^{y_{i}-1} \frac{1}{j+\mu_{i} / c_{i}}\right\} \frac{\mu_{i}\left(c_{i}+1\right)\left(c_{i}+2\right)}{c_{i}^{3}}-\frac{y_{i}-\mu_{i}}{c_{i}^{2}}\left(c_{i}+1\right)\right], \\
\frac{\partial^{2} l}{\partial \tau \partial \lambda}= & \sum_{i=1}^{N} \frac{\partial^{2} l_{i}}{\partial \tau \partial \lambda}=\sum_{i=1}^{N} \frac{\partial^{2} l_{i}}{\partial \tau^{2}} \log \left(\mu_{i}\right),
\end{aligned}
$$

and

$$
\frac{\partial^{2} l}{\partial \lambda^{2}}=\sum_{i=1}^{N} \frac{\partial^{2} l_{i}}{\partial \lambda^{2}}=\sum_{i=1}^{N} \frac{\partial^{2} l_{i}}{\partial \tau^{2}}\left(\log \left(\mu_{i}\right)\right)^{2} .
$$

The Fisher information can be calculated by replacing $\sum_{j=0}^{y_{i}-1} 1 /\left(j+\mu_{i} / c_{i}\right)$ and $\sum_{j=0}^{y_{i}-1} 1 /\left(j+\mu_{i} / c_{i}\right)^{2}$ by $\sum_{k=1}^{\infty}\left\{\sum_{j=0}^{k-1} 1 /\left(j+\mu_{i} / c_{i}\right)\right\} \operatorname{Pr}(y=k)$ and $\sum_{k=1}^{\infty}\left\{\sum_{j=0}^{k-1} 1 /\left(j+\mu_{i} / c_{i}\right)^{2}\right\} \operatorname{Pr}(y$ $=k$ ), respectively, where $\operatorname{Pr}($.$) is the NB-PVF probability. The asymptotic$ variance-covariance estimate of the parameter estimate from the NB-PVF model can be calculated by inverting the Fisher information matrix.

\section{Quasilikelihood/pseudolikelihood estimating equations}

In general, when the distribution of the data is complicated or the specification of the distribution is difficult, it is appropriate to apply the quasilikelihood method. Another merit of the quasilikelihood method is that it is robust and can be implemented by a GLIM program.

Now, from the mean-variance relationship (1), Wedderburn's (1974) quasilikelihood estimating equation is

$$
U_{1}(\beta)=\sum_{i=1}^{N} \frac{y_{i}-\mu_{i}}{V_{i}(\beta, \theta)} \frac{\partial \mu_{i}}{\partial \beta}=0 .
$$

where $\theta=(\log (\phi), \lambda)=(\tau, \lambda)$, and $V_{i}(\beta, \theta)=\phi \mu_{i}^{\lambda}$. The variance function would be appropriate for gamma or lognormal data when $\lambda=2$, and when $\lambda=1$, it 
would be appropriate for the Poisson data. In radioimmunoassay data, $\lambda$ is often in the range of 1.2 to 1.6 (Carroll and Ruppert 1988, p. 5). This equation can be solved by a GLIM program if $\theta=(\tau, \lambda)$ is known. We note that even if the variance function is misspecified the mean parameter estimate from equation (8) is still consistent and $\sqrt{N}\left(\beta-\beta_{0}\right)$ has an asymptotic normal distribution where $\beta_{0}$ is the true mean parameter. This fact is known for a more general framework (Liang and Zeger 1986). A component of deviance as a measure of the goodness of fit of the mean model can be given by

$$
2 \int_{\mu}^{y} \frac{y-t}{\phi t^{2}} d t=\frac{2}{\phi(1-\lambda)} y\left(y^{1-2}-\mu^{1-2}\right)-\frac{2}{\phi(2-\lambda)}\left(y^{2-2}-\mu^{2-2}\right) .
$$

In this model, the variance function $V_{i}=V_{i}(\beta, \theta)$ includes the unknown parameter $\theta=(\log (\phi), \lambda)$. Noting that $V_{i}$ can be written in a $\log$-linear form in terms of $\tau=\log (\phi)$ and $\lambda$, to estimate $\theta$, we can construct a quasilikelihood estimating equation for $Z_{i}=\left(y_{i}-\mu_{i}\right)^{2}$. The mean and variance of $Z_{i}$ is given by

$$
\left\{\begin{array}{l}
E\left(Z_{i}\right)=V_{i}, \\
\operatorname{Var}\left(Z_{i}\right)=2 V_{i}^{2}\left(1+\gamma_{2 i} / 2\right),
\end{array}\right.
$$

where $\gamma_{2 i}$ is the kurtosis of $y_{i}$. However, $\gamma_{2 i}$ is in general unknown. Therefore, we use $2 V_{i}^{2}$ as a variance function of $Z_{i}$. The following pseudolikelihood estimating equation is thus obtained (Carroll and Ruppert 1982),

$$
U_{2}(\theta)=\sum_{i=1}^{N} \frac{Z_{i}-V_{i}}{2 V_{i}^{2}} \frac{\partial V_{i}}{\partial \theta}=0,
$$

where $\theta=(\tau, \theta)=(\log (\phi), \lambda)$. This pseudolikelihood estimating equation is an approximate quasilikelihood estimating equation for $\theta$. The equation can be solved by using the generalized least square, which is the method of estimation for generalized linear models; this estimating equation can be fit by GLIM as a log link gamma model with scale factor 2 .

Convergence would be obtained by iterating back and forth the applications of the quasilikelihood and pseudolikelihood estimating equations, (8) and (11). This estimate is called a QL/PL estimate. The criteria of the convergence is measured by the following Carroll and Ruppert's pseudolikelihood (1982),

$$
-2 l_{P L}=\sum_{i=1}^{N}\left\{\log \left(V_{i}(\beta, \theta)\right)+\frac{Z_{i}}{V_{i}(\beta, \theta)}\right\} .
$$

This pseudolikelihood corresponds to the deviance of the estimating equation (11). The solution, $\bar{\theta}$, of equation (11) thus attained the minimum of the pseudolikelihood, $-2 l_{P I}$. The above derivations of the pseudolikelihood estimating equation and the pseudolikelihood are somewhat different from those of Carroll and Ruppert (1988). We call the method of the analysis using the estimating equations (8) and (11) a quasilikelihood/pseudolikelihood (QL/PL) method (Breslow 1990b).

To test the hypothesis $\tau=\log (\phi)=0$ i.e., $\phi=1$, we can exploit the Wald test for which robust standard errors are given by the robust version of the 
formulae (15) and (16) described below, or the approximate pseudolikelihood ratio test statistic (Carroll and Ruppert 1988),

$$
L R_{P}=\left\{2 l_{P \mathbf{P}}(\bar{\beta}, \bar{\phi}, \bar{\lambda})-2 l_{P \mathbf{P}}\left(\beta^{*}, \phi=1, \lambda^{*}\right)\right\},
$$

where $\beta^{*}$ and $\lambda^{*}$ are the maximum QL/PL estimates at $\phi=1$. The approximate modified pseudolikelihood ratio test statistic, $L R_{P}^{*}$, is derived by multiplying (13) by a factor of $2 /\left(2+\bar{\gamma}_{2}\right)$, where $\bar{\gamma}_{2}$ is the average kurtosis of $y_{i}$ in fitting the full model. If $N$ is large, $L R_{P}$ and $L R_{P}^{*}$ are approximately asymptotically distributed as $\chi_{1}^{2}$. For the test of $\lambda=1$, we can construct a similar pseudolikelihood ratio test.

From the theory of unbiased estimating equations, e.g., Inagaki (1973), for $U=\left(U_{1}^{T}, U_{2}^{T}\right)^{T}$ with respect to $\omega=(\beta, \theta)$, under some regularity conditions, assuming that $\left(\beta_{0}, \theta_{0}\right)$ be the true parameter, $\sqrt{N}\left(\bar{\beta}-\beta_{0}, \bar{\theta}-\theta_{0}\right)$ converges in law to the multivariate normal distribution with mean zero and variancecovariance matrix,

$$
\Gamma^{-1} \Lambda\left(\Gamma^{-1}\right)^{T}
$$

where

$$
\Gamma=\lim _{N \rightarrow \infty} \frac{1}{N} E\left(\frac{\partial U}{\partial \omega^{T}}\right)=\left(\begin{array}{cc}
\Gamma_{11} & 0 \\
\Gamma_{21} & \Gamma_{22}
\end{array}\right) \text { and } \Lambda=\lim _{N \rightarrow \infty} \frac{1}{N} \operatorname{Cov}(U)=\left(\begin{array}{ll}
\Lambda_{11} & \Lambda_{12} \\
\Lambda_{21} & \Lambda_{22}
\end{array}\right),
$$

where

$$
\begin{aligned}
& \Gamma_{11}=\lim _{N \rightarrow \infty}-\frac{1}{N} \sum_{i=1}^{N} \frac{1}{V_{i}} \frac{\partial \mu_{i}}{\partial \beta} \frac{\partial \mu_{i}}{\partial \beta^{T}}, \quad \Gamma_{21}=\lim _{N \rightarrow \infty}-\frac{1}{N} \sum_{i=1}^{N} \frac{1}{V_{i}^{2}} \frac{\partial V_{i}}{\partial \theta} \frac{\partial V_{i}}{\partial \beta^{T}}, \\
& \Gamma_{22}=\lim _{N \rightarrow \infty}-\frac{1}{N} \sum_{i=1}^{N} \frac{1}{V_{i}^{2}} \frac{\partial V_{i}}{\partial \theta} \frac{\partial V_{i}}{\partial \theta^{T}},
\end{aligned}
$$

and

$$
\begin{aligned}
& \Lambda_{11}=\lim _{N \rightarrow \infty} \frac{1}{N} \sum_{i=1}^{N} \frac{1}{V_{i}} \frac{\partial \mu_{i}}{\partial \beta} \frac{\partial \mu_{i}}{\partial \beta^{T}}=-\Gamma_{11}, \\
& \Lambda_{21}=\lim _{N \rightarrow \infty} \frac{1}{N} \sum_{i=1}^{N} \frac{\mu_{8 i}}{V_{i}^{3}} \frac{\partial V_{i}}{\partial \theta} \frac{\partial \mu_{i}}{\partial \beta^{T}}=\lim _{N \rightarrow \infty} \frac{1}{N} \sum_{i=1}^{N} \frac{\gamma_{1 i}}{V_{i}^{3 / 2}} \frac{\partial V_{i}}{\partial \theta} \frac{\partial \mu_{i}}{\partial \beta^{T}}=\Lambda_{12}^{T}, \\
& \Lambda_{22}=\lim _{N \rightarrow \infty} \frac{1}{N} \sum_{i=1}^{N} \frac{\mu_{4 i}-V_{i}^{2}}{V_{i}^{4}} \frac{\partial V_{i}}{\partial \theta} \frac{\partial V_{i}}{\partial \theta^{T}}=\lim _{N \rightarrow \infty} \frac{1}{N} \sum_{i=1}^{N} \frac{2+\gamma_{2 i}}{V_{i}^{2}} \frac{\partial V_{i}}{\partial \theta} \frac{\partial V_{i}}{\partial \theta^{T}},
\end{aligned}
$$

where $\gamma_{1 i}$ and $\gamma_{2 i}$ are the skewness and kurtosis of $y_{i}, \gamma_{1 i}=\left(y_{i}-\mu_{i}\right)^{3} / V_{i}^{3 / 2}$ and $\gamma_{2 i}=\left(y_{i}-\mu_{i}\right)^{4} / V_{i}^{2}-3$ for the robust variance estimate, and $\gamma_{1 i}=\left(V_{i}^{2} / \mu_{i}-1\right) \mid$ $\sqrt{V_{i}}$ and $\gamma_{2 i}=6\left(V_{i}^{2}-\mu_{i}\right) / \mu_{i}^{2}+1 / V_{i}^{2}$ for NB-PVF.

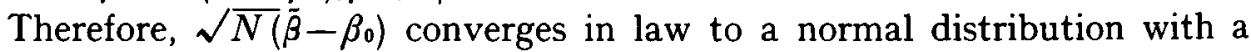
mean zero and variance-covariance matrix,

$$
A_{11}^{-1}=\left(\lim _{N \rightarrow \infty} \frac{1}{N} \sum_{i=1}^{N} \frac{1}{V_{i}} \frac{\partial \mu_{i}}{\partial \beta} \frac{\partial \mu_{i}}{\partial \beta^{T}}\right)^{-1},
$$

which is the same as the model-based variance from a single estimating equation (8) if $\theta$ is known. This means that the estimation of the mean parameter $\beta$ is not affected by the estimation of variance parameter $\theta$. And $\left.\sqrt{N(} \tilde{\theta}-\theta_{0}\right)$ converges in law to bivariate normal distribution with a mean zero and vari- 
ance-covariance matrix,

$$
\Gamma_{22}^{-1}\left(\Lambda_{22}-\Gamma_{21} \Gamma_{11}^{-1} \Lambda_{12}-\Lambda_{21} \Gamma_{11}^{-1} \Gamma_{21}^{T}+\Gamma_{21} \Lambda_{11}^{-1} \Gamma_{21}^{T}\right) \Gamma_{22}^{-1} .
$$

$\Gamma_{22}^{-1} \Lambda_{22} \Gamma_{22}^{-1}$ is the variance-covariance estimate from a single estimating equation (11) for the variance parameter $\theta$. This means that the estimation of variance parameter $\theta$ is affected by the estimation of mean parameter $\beta$.

\section{Efficiency comparison of the two estimation methods}

We calculated the efficiency of the QL/PL estimate under the assumption that the data are distributed as NB-PVF. The variance estimates for finite $N$ are obtained by inserting parameter estimates into (14) with "lim" omitted in all expressions. The Fisher information can also be calculated for finite $N$. Asymptotic relative efficiency (ARE) of the estimates $(\bar{\beta}, \bar{\tau}, \bar{\lambda})$ from the QL/PL estimating equation relative to the maximum likelihood estimates $(\hat{\beta}, \hat{\tau}, \hat{\lambda})$ are obtained by calculating $\operatorname{ARE}(\bar{\beta})=\operatorname{avar}(\sqrt{N}(\hat{\beta}-\beta)) / \operatorname{avar}(\sqrt{N}(\bar{\beta}-\beta))$, $\operatorname{ARE}(\bar{\tau})=\operatorname{avar}(\sqrt{N}(\hat{\tau}-\tau)) / \operatorname{avar}(\sqrt{N}(\bar{\tau}-\tau))$ and $\operatorname{ARE}(\bar{\lambda})=\operatorname{avar}(\sqrt{N}(\hat{\lambda}-\lambda)) /$ $\operatorname{avar}(\sqrt{N}(\bar{\lambda}-\lambda))$ and then comparing the inverse Fisher information matrix of the NB-PVF model with (15) and (16) in which $\gamma_{1 i}$ and $\gamma_{2 i}$ from the NBPVF model are used. Table 1 shows the efficiency for various values of each parameter in which $x_{i}$ takes $1 / 3$ of 1,2 and $3 . \quad \tilde{\beta}$ is fully efficient in all situations, which is also true for the standard negative-binomial model (Lawless 1987). When $\lambda$ is close to one, e.g., $\lambda=1.1$, the efficiency of $\bar{\lambda}$ is more than 0.9 . When $\lambda$ is about 1.5 , the efficiency of $\tilde{\lambda}$ is still not poor. It was observed that, in the NB-PVF model, the mean parameter estimates and the variance parameter estimates have approximately no correlation in our situation, i.e., $|\rho|<0.05$, which means that they are approximately independent.

Table 1. Asymptotic relative efficiency (ARE) of the QL/PL estimates

\begin{tabular}{rccccc}
\hline$\beta$ & $\tau$ & $\lambda$ & ARE $(\tilde{\beta})$ & ARE $(\tilde{\tau})$ & ARE $(\tilde{\lambda})$ \\
\hline 20 & 0.0 & 1.1 & 1.000 & 0.900 & 0.909 \\
20 & 0.0 & 1.5 & 1.000 & 0.620 & 0.628 \\
20 & 0.0 & 2.0 & 1.000 & 0.279 & 0.271 \\
200 & 0.0 & 1.1 & 1.000 & 0.981 & 0.982 \\
200 & 0.0 & 1.5 & 1.000 & 0.769 & 0.775 \\
200 & 0.0 & 2.0 & 1.000 & 0.208 & 0.207 \\
\hline
\end{tabular}

\section{Example}

Using data collected from several experiments conducted during the preceding 20 years, Armitage (1957) investigated the relationship of the observed mean and the variance and found that the variance of the pock count shows a far excess from the average pock count. He stated that this extra-Poisson variability can not be formulated by the quadratic function of the mean, which is the variance of the standard negative-binomial model (Lawless 1987), but 
can be formulated by a power of the mean. There was no finding, however, as to how the pock counts were distributed. A type of variation was also observed with counts of reverse mutant colonies from the Ames/salmonella assay (Myers et al. 1981).

A systematic study of the variability of lesion counts produced on the chorioallantoic membranes of chick embryos by viruses of the pox group was made (Armitage 1957). Such pock counts were widely made to investigate "the time of appearance and persistence of antibody after vaccination and in smallpox patients" (McCarthy et al. 1958).

Table 2 and Figure 1 shows the data from a titration experiment made by Armitage and his colleagues (McCarthy et al. 1958). Let $y_{k j}, j=1, \ldots, n_{k}=8$ or $10 ; k=1, \ldots, K=6$, be an individual count for the $j$-th replication at the $k$-th dilution, and $\mu_{k}=E\left(y_{k j}\right)$ and $V_{k}=\operatorname{Var}\left(y_{k j}\right)$. Figure 2 shows the relation

Table 2. Titration of twofold dilution of variola virus (McCarthy et al. 1958, Table 1)

\begin{tabular}{clcrcr}
\hline Dilution Factor $2^{k-1}$ & Individual membrane counts $\left\{y_{k j}\right\}$ & $n_{k}$ & mean $^{1)}$ & variance $^{1)}$ \\
\hline 1 & $116,151,171,194,196,198,208,259$ & 8 & 186.625 & 1781.1 \\
2 & $71,74,79,93,94,115,121,123,135,142$ & 10 & 104.7 & 667.3 \\
4 & $27,33,34,44,49,51,52,59,67,92$ & 10 & 50.8 & 360.4 \\
8 & $8,10,15,22,26,27,30,41,44,48$ & 10 & 27.1 & 195.0 \\
16 & $5,6,9,11,13,15,16,19,20,22$ & 10 & 13.6 & 34.3 \\
32 & $6,6,7,7,8,9,9,9,11,20$ & 10 & 4.1 & 16.8 \\
\hline
\end{tabular}

1) Observed value.

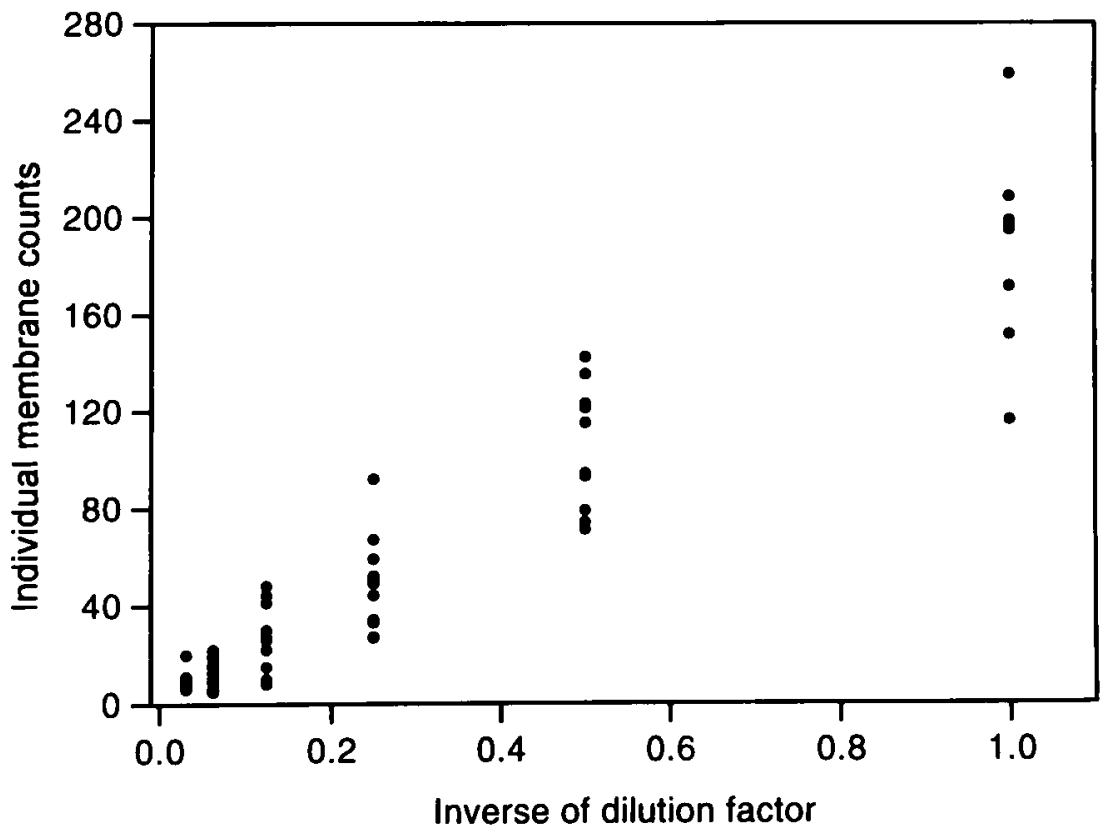

Figure 1. Pock count data from McCarthy et al. (1958). 




Figure 2. The log of observed variance and the log of observed mean for pock count data.

Table 3. Parameter estimates from negative binomial maximum likelihood method and Quasilikelihood/pseudolikelihood method

\begin{tabular}{|c|c|c|c|c|}
\hline \multirow[t]{2}{*}{ Parameter } & \multicolumn{2}{|c|}{$\begin{array}{l}\text { Negative binomial maximum } \\
\text { likelihood method } 1)\end{array}$} & \multicolumn{2}{|c|}{$\begin{array}{l}\text { Quasilikelihood/pseudolikelihood } \\
\text { method }\end{array}$} \\
\hline & \multicolumn{4}{|c|}{ Mean model: $\mu=\beta x$} \\
\hline$\beta$ & 208.478 & 207.795 & 204.610 & 207.897 \\
\hline \multirow[t]{2}{*}{ s.e. } & 10.250 & 9.516 & 9.036 & 9.472 \\
\hline & & Varian & odel: $V=\phi \mu^{\lambda}$ & \\
\hline $\log (\phi)$ & -0.122 & 0.0 & 0.679 & 0.0 \\
\hline s.e. & 0.623 & - & 0.595 & - \\
\hline$\lambda$ & 1.487 & 1.454 & 1.283 & 1.457 \\
\hline s.e. & 0.175 & 0.051 & 0.160 & 0.050 \\
\hline Pearson $\chi^{2}$ & 62.617 & 62.043 & 58.000 & 61.494 \\
\hline $\mathrm{LL}$ or $\left.-2 \mathrm{PL}^{2}\right)$ & 12648.992 & 12648.974 & 359.870 & 360.996 \\
\hline
\end{tabular}

1) Newton-Raphson method; 2) Log of maximum likelihood or -2 times Pseudolikelihood.

of $\log$ of observed means and $\log$ of observed variances by each titration level. It is clear that the observed variance is proportional to a power of the mean. The two figures suggest model (1), in which $x$ is reciprocal of the dilution factor. Breslow (1990a) made an analysis of this pock count data using the QL/PL method. We show parameter estimates using two methods, the negativebinomial likelihood method and the QL/PL method using $U_{1}$ and $U_{2}$, in Table 
3. Both methods resulted in similar parameter estimates. In order to obtain the result of the maximum likelihood method, we tested the hypothesis for $\lambda=1$ at $\phi=1$, a Poisson hypothesis, and found it to be significant $(p<0.000)$ by the Wald test. For the QL/PL method, the robust standard errors for the parameters $\tau$ and $\lambda$ using (16) are 0.796 and 0.200 , respectively in the full model. In the Wald test, the hypothesis $\tau=0$ was not rejected $(p=0.199)$ and the hypothesis $\lambda=1$, which is a Poisson hypothesis, was suggestive $(p=0.081)$. The approximate pseudolikelihood ratio test for $\tau=0$ was not significant $(p=0.289)$.

\section{Discussion}

Because the negative-binomial model with power variance function was derived using mathematical flexibility, that is, because the mean-variance relation was simply embedded in a negative binomial model, this model would not be appropriate for the pock count data set. The QL/PL estimating equation approach assumes only the first two moments and a mean variance relationship; the QL/PL method, which is a semiparametric method, therefore, is robust since we do not assume the higher order moments. Furthermore, in our power variance function situation, the mean parameter estimation using the QL/PL method was almost fully efficient.

Firth (1987) investigated the ARE in various situations, under a constant coefficient of variation model in the continuous data and under a McCullagh and Nelder type overdispersion model (McCullagh and Nelder 1989) in which $\operatorname{Var}(y)=\sigma^{2} V(\mu)$ where $\sigma^{2}$ is a overdispersion parameter and $V(\mu)$ is a known variance function of mean $\mu$. He concluded that under these conditions the ARE of quasilikelihood estimate is not deteriorated if the coefficient of variation parameter or the overdispersion parameter is mild, and further suggested a quadratic estimating equation. Our variance model, however, is not in the range of Firth's assumption. In our situation, the ARE of QL/PL estimate of $\lambda$ becomes lower when $\lambda$ becomes larger. In practice, $\lambda$ is often near 1.5 and ARE at $\lambda=1.5$ is not poor. With a count data of $\lambda=2.0$, the ARE of the QL/ PL estimate of $\lambda$ is low even though the ARE of the QL/PL estimate of $\beta$ is still fully efficient. In such a case, the NB-PVF model would be appropriate. We neglect the kurtosis of $y_{i}$ in the pseudolikelihood estimating equation. In order to adjust the kurtosis in the equation, however, we can put $\gamma_{2 i}$ as a grouped average kurtosis or NB-PVF kurtosis. This provides a more efficient estimate of $\theta$.

From $a_{i}>0$ in equations (7), the range of parameters $\lambda$ and $\phi$ in NB-PVF is restricted by the inequalities $\phi \mu_{i}^{i}>\mu_{i} ; i=1, \ldots, N$. This means that NBPVF is appropriate for extra-Poisson data or overdispersed count data. In this range of $\lambda$ and $\phi$, when we use QL/PL method, we can assume NB-PVF as a background extra-Poisson model.

In $\mathrm{ED}^{(r)}$-PVF, the score equation for the mean parameters is a quasilikelihood estimating equation when power $r$ is fixed. The score equation for $r$, however, is very complicated and to implement the likelihood method for $r$ is sometimes difficult. Also, the assumption that the real data belongs to this 
distribution is difficult to verify. Therefore, the pseudolikelihood estimating equation for the estimation of the power $r$ described in this report could be useful for these situations and may sometimes be superior to the maximum likelihood method in certain cases of practical data analysis.

\section{Acknowledgements}

The author wishes to thank the referees for their constructive comments.

\section{REFERENCES}

[1] Armitage, P. (1957). Studies in the variability of pock counts. J. of Hygiene 55, $564-581$.

[2] Baker, R. J. and Nelder, J. A. (1978). The GLIM manual release 3. Numerical Algorithm Group, Oxford.

[ 3 ] Breslow, N. E. (1990a). Further studies in the variability of pock counts. Statist. in Med. 9, 615-626.

[4] Breslow, N. E. (1990b). Tests of hypothesis in overdispersed poisson regression and other quasi-likelihood models. J. Amer. Statist. Assoc. 85, 565-571.

[ 5 ] Carroll, R. J. and Ruppert, D. (1982). Robust estimation in heteroscedastic linear models. Annal. Statist. 10, 429-442.

[6] Carroll, R. J. and Ruppert, D. (1988). Transformation and weighting in regression. Chapman and Hall, London.

[ 7 ] Cox, D. R. and Reid, N. (1987). Parameter orthogonality and approximate conditional inference. J.R. Statist. Soc. B 49, 1-39.

[ 8 ] Davidian, M. and Carroll, R. J. (1988). A note on extended quasilikelihood. J. $R$. Statist. Soc. B 50, 74-82.

[9] Davidian, M. and Carroll, R. J. (1987). Variance function estimation. J. Amer. Statist. Assoc. 82, 1079-1091.

[10] Firth, D. (1987). On the efficiency of quasi-likelihood estimation. Biometrika 74, 233-243.

[11] Fisher, R. A. (1941). The negative-binomial distribution. Ann. Eug. 11, 192-197.

[12] Fisher, R. A. (1953). Note on the efficient fitting of the negative-binomial. Biometrics 9, 197-200.

[13] Inagaki, N. (1973). Asymptotic relations between the likelihood estimating function and the maximum likelihood estimator. Annal. Inst. Statist. Math. 25, 1-26.

[14] Jorgensen, B. (1987) Exponential dispersion model. J. R. Statist. Soc. B 49, 127172.

[15] Kupper, L. L., Portier, C., Hogan, M. D. and Yamamoto, E. (1986). The impact of litter effects on dose response modeling in teratology. Biometrics 42, 85-98.

[16] Lawless, J. F. (1987). Negative binomial and mixed Poisson regression. Can. J. Statist. 15, 209-225.

[17] Liang, K. Y. and Zeger, S. L. (1986). Longitudinal data analysis using generalized linear models. Biometrika 73, 13-22.

[18] McCarthy, K., Downie, A. W. and Armitage, P. (1958). The antibody response in man following infection with viruses of pox group: I. An evaluation of the pock counting method for measuring neutralizing antibody. J. Hygiene 56, 84-100.

[19] McCullagh, P. and Nelder, J. A. (1989). Generalized linear models. Second ed. Chapman and Hall, London.

[20] Myers, L. E., Sexton, N. H., Southerland, L. I. and Wolff, T. J. (1981). Regression analysis of Ames test data. Environmental Mutagenesis 3, 575-586.

[21] Nishii, R. (1992). Box-Cox transformation and power variance functions in exponential dispersion model. Research Memorandum, Institute of Statistical Mathematics, Tokyo.

[22] Wedderburn, R. W. M. (1974). Quasilikelihood functions, generalized linear models, 
and Gauss-Newton method. Biometrika 61, 439-449.

[23] Yamamoto, E. and Yanagimoto, T. (1988). Litter effects to dose-response curve estimation. J. Japan Statist. Soc. 18, 97-106. 